\title{
Jet Shapes at CMS
}

\author{
Pelin Kurt* \\ Cukurova University, Adana, 01330 TURKEY. \\ E-mail: pkurtecern. $\mathrm{ch}^{\dagger}$
}

\section{On behalf of the CMS Collaboration}

The CMS (Compact Muon Solenoid) detector will observe high transverse momentum jets produced in the final state of proton-proton collisions at the center of mass energy of $14 \mathrm{TeV}$. These data will allow us to measure jet shapes, defined as the fractional transverse momentum distribution as a function of the distance from the jet axis. Since jet shapes are sensitive to parton showering processes they provide a good test of Monte Carlo event simulation programs. In this note we present a study of jet shapes reconstructed using calorimeter energies where the statistics of all distributions correspond to a CMS data set with $10 \mathrm{pb}^{-1}$ of integrated luminosity. We compare the predictions of the Monte Carlo generators PYTHIA and HERWIG++.

2008 Physics at LHC

September 29 - 4 October 2008

Split, Croatia

\footnotetext{
* Speaker.

†The author would like to thank to the CMS QCD Group especially to Anwar Bhatti, Marek Zielinski, Nikos Varelas and Klaus Rabbertz for their active contributions to the presented analysis, related proceeding and given talk.
} 


\section{Introduction}

The transverse momentum profile of a jet, called jet shape [1] is sensitive to multi parton emissions from the primary outgoing parton and provides a good test of parton showering description by Quantum Chromodynamics (QCD), the theory of strong interactions. Jet shapes have been used to test perturbative QCD (pQCD) calculations [2]. Jet shapes are also sensitive to jet clustering, underlying event contribution and hadronization effects, and can be used to tune MC generators. The Compact Muon Solenoid (CMS) [3] detector is a multipurpose apparatus, designed to explore the physics at the $\mathrm{TeV}$ energy scale at the Large Hadron Collider (LHC). Here, we present a study of jet shapes, and compare the results obtained with various MC generators. The sensitivities of jet shapes to the underlying event (UE) model and to the flavor of the initiating parton are also explored. QCD dijet events were generated with PYTHIA tunings DWT [4] and DW [5], ALPGEN [6], and HERWIG++ [7, 8]. For multiple-parton interaction and underlying event different tunings were used for both PYTHIA and HERWIG. Results are presented as expected for $p p$ collisions at $14 \mathrm{TeV}$ assuming an integrated luminosity of $\int L d t=10 \mathrm{pb}^{-1}$. In this analysis we use jets reconstructed with the Seedless Infrared Safe Cone algorithm [9] of radius $R=\sqrt{(\Delta y)^{2}+(\Delta \phi)^{2}}$ in $(y, \phi)$ space $(R=0.7)$, where $\Delta y$ and $\Delta \phi$ specify the cone dimensions in rapidity [10] and azimuth respectively. The jet shape is defined as the average fraction of the jet transverse momentum within a cone of a given size $r$ around the jet axis, using all particles and calorimeter towers. Only the two leading jets within $|y|<1$ are considered. The integrated jet shapes are defined as:

$$
\psi(r)=\frac{1}{N_{\text {jets }}} \sum_{\text {jets }} \frac{P_{T}(0, r)}{P_{T}(0, R)}
$$

where $P_{T}(0, r)$ is the scalar sum of transverse momenta of all $\mathrm{MC}$ truth particles within the distance $r$ from the jet axis with $\psi(r=R)=1.0$. Due to various detector effects, the measured (calorimeter) jet shapes are different than the true (particle) jet shapes. The correction factors are calculated from the momentum fractions of the MC events before and after the CMS detector simulation. Corrected jet shapes agree with particle jets by construction. The jet shape $\psi(r)$ increases faster with $r$ for jets at larger $P_{T}$ indicating that larger $P_{T}$ jets are more collimated [11]. We tested the correction derived from PYTHIA on an independent sample generated using ALPGEN. The correction factors determined from PYTHIA events work reasonably well for ALPGEN, as expected [11]. To determine the sensitivity of jet shapes to the UE contribution, event samples were generated using PYTHIA DW which has a less enhanced UE contribution than PYTHIA with tune DWT. A difference in jet shapes due to the UE contribution is visible at low jet $P_{T}$ only [11]. PYTHIA DW tends to produce narrower jet shapes in the low $P_{T}$ region. Jet shapes are also sensitive to fragmentation effects since the calorimeter response depends on the energies of the particles in the jets. The contributions of different models of the jet shape was investigated by comparing particle level jet shapes in PYTHIA DWT and HERWIG++ 2.2. Their observed difference is less than 5\% at $r<0.3$ and decreases as a function of $r$ [11]. The jet shapes are also sensitive to quark and gluon jet contributions. Using parton information from PYTHIA we classify hadron level jets as initiated by a quark or gluon. Fig. ?? presents the $P_{T}$ fraction contained in an annulus $0.2<r<0.7$ as a function of the jet $P_{T}$. Reconstructed calorimeter jets from the full CMS Monte Carlo simulation are compared with parton shower MC predictions for quark and gluon jets. Several sources of 
systematic uncertainties were investigated [11], namely those from jet energy calibration [12], jet fragmentation, calorimeter response and transverse showering, as a function of jet $P_{T}$ and distance from the jet axis $r$. The systematic uncertainty is dominated by the overall jet energy scale, jet fragmentation and calorimeter simulation uncertainties. The total systematic uncertainty at $r=0.2$ is $12 \%$ at $P_{T}=60 \mathrm{GeV}$, decreasing to $4 \%$ at jet $P_{T}=1 \mathrm{TeV}$.

Using PYTHIA and HERWIG++ MC simulations, we have investigated a technique to measure jet shapes in $p p$ collisions for the two leading jets in the kinematic region $60 \mathrm{GeV}<P_{T}<1.4$ $\mathrm{TeV}$ and $|y|<1$. As expected in QCD, jet shapes are observed to become narrower at increasing jet $P_{T}$. QCD predicts different shapes of jets originating from quarks and gluons. A measurement of the jet shapes in the context of the PYTHIA Monte Carlo gives an estimate of the fraction of gluon initiated jets in data as a functionof jet $P_{T}$.

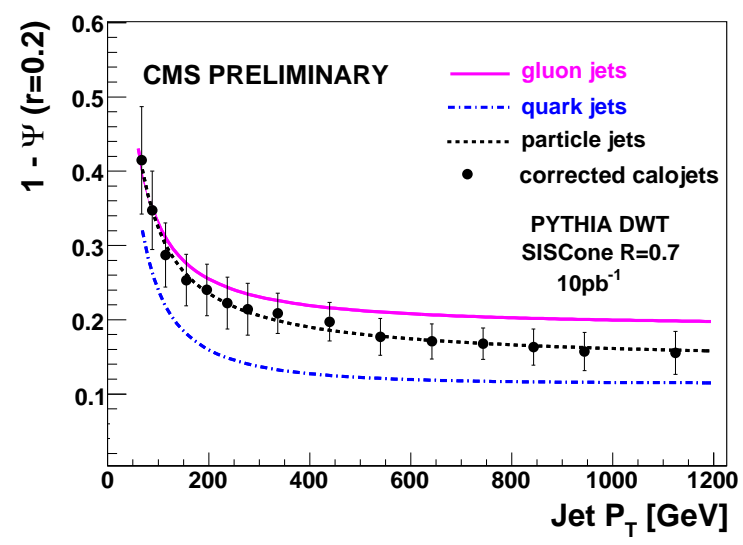

\begin{abstract}
Figure 1: The fractional transverse momentum of a jet in an annulus $0.2<r<$ 0.7 as a function of the jet $P_{T}$ in the rapidity region $|y|<1$. The reconstructed calorimeter jets, from PYTHIA Tune DWT (black points) are shown along with PYTHIA predictions for quark (dashed-dotted line) and gluon initiated jets (solid line), and for all particle jets (dashed line). Systematic and statistical errors for the reconstructed calorimeter jets are added in quadrature.
\end{abstract}

\title{
References
}

[1] S. D. Ellis, Z. Kunszt, and D. E. Soper, Phys. Rev. Lett. 69 (1992), M. H. Seymour, Nucl. Phys. B513 (1998).

[2] CDF Collaboration, F. Abe et al., Phys. Rev. Lett. 70 (1993); CDF Collaboration A. Abulencia et al., Phys. Rev. D78:072005 (2008); D0 Collaboration, S. Abachi et al., Phys. Lett. B357; CDF Collaboration, D. E. Acosta et al.,Phys. Rev. D71 (2005) 112002; H1 Collaboration, C. Adloff et al., Eur. Phys. J. C12 (2000); ZEUS Collaboration, J. Breitweg et al., Eur. Phys. J. C8 (1999)

[3] The CMS Collaboration, S Chatrchyan et al 2008 JINST 3 S08004.

[4] T. Sjostrand, S. Mrenna, and P. Skands, PYTHIA 6.4, JHEP 05 (2006) 026.

[5] The CMS Collaboration, CMS-Note-2006/067.

[6] M. L. Mangano et al., ALPGEN, JHEP 07 (2003) 001.

[7] M. Bahr et al., Herwig++ 2.2, arXiv:0804.3053.

[8] M. H. Seymour et al., arXiv:0809.2669 and JHEP 07 (2008) 076.

[9] G. P. Salam and G. Soyez, JHEP 05 (2007) 086.

[10] The rapidity is defined as $y=\frac{1}{2} \log \frac{E+P_{Z}}{E-P_{Z}}$, where $E$ denotes the energy and $P_{Z}$ is the $z$ component of the momentum.

[11] The CMS Collaboration, CMS-PAS QCD-08-005 .

[12] The CMS Collaboration, CMS-PAS JME-007-002. 